\title{
Energy-efficient inverter circuit for the low- voltage asynchronous driver controller with autonomous power supply
}

\author{
Alexander Boldyrev* \\ Don State Technical University, Rostov-on-Don, Russian Federation
}

\begin{abstract}
The design of the energy-efficient inverter circuit for the asynchronous frequency-controlled three-phase electric driver with autonomous low-voltage DC power supply. The components analysis of the electric drive, the main components selection approach: microcontroller, three-phase driver and power transistors. R\&D prototype of the energy-efficient inverter circuit was created, characterized by an average consumption current reduced by $5 \%$. Reducing energy losses is achieved by reducing dynamic losses in the power circuits of a low-voltage inverter and using several circuit solutions.
\end{abstract}

\section{Introduction}

One of the focus areas in energy-efficient technologies research and development is in characteristics fine tuning and identifying energy efficient spots, to provide effective and efficient control system algorithms for the electric drivers.

Thanks to the semiconductor industry continuous improvements, particularly power electronics and microcontrollers, the variable speed drivers improved their usability, applicability and eventually reduced the costs of the complete solutions. Thus, there are following advantages due to the advanced control algorithms used jointly with the microcontrollers:

- Improved system energy efficiency - speed control lowers down power losses of the motor;

- Performance enhancements - due to the digital control such features like intelligent closed loops, frequency properties adjustments covering the potential failure ranges and improved system integration, can be added;

- Simplification of electromechanical energy conversion - variable drivers eliminate the need for transmissions, gearboxes;

- Simple software upgrade process - flash memory-based microcontrollers software can be upgraded with minimum down time, through the software updates of their algorithms and respective variables.

It is known that using variable drivers the complexity of the system can grow too, however the IC yield and manageable design allows one to have predictable reliability and

\footnotetext{
*Corresponding author: avb49@aaanet.ru
} 
cost of the system. In number of cases, the total cost is in fact close to the cost of the fix speed driver.

\section{Components of the asynchronous electric drive with autonomous power supply}

Interesting case is in asynchronous three-phase variable speed drivers with autonomous (for example, battery) power supply, which are low-voltage types by default. In addition to the low-voltage induction motor, the control system includes the following [1]:

- microcontroller (MC);

- three-phase driver;

- feedback sensors;

- opto-isolation between the power circuits and the microcontroller;

- power supply.

Most of the listed components are easily adaptable to low supply voltages. The only parts need adaptation are microcircuits of three-phase drivers, initially designed by default for the high voltage power supplies. This and several other issues were resolved during the development and testing of a self-powered inverter for controlling a low-voltage multifunctional asynchronous electric driver.

\subsection{Microcontroller}

Specialized microcircuit MC3PHAC (Motorola), the inverter controller is considered as the base for the exemplary design. MC3PHAC is a microcontroller with ready to use software stack for application areas of control systems for the asynchronous variable frequency drives $[2,3]$. The microcircuit software cannot be modified; adaptation of the control algorithm for the specific type of electric driver is carried out by changing the numerical constants. Constant's settings are possible in two ways: by potentiometers on the controller board of the control unit or from a higher-level computer connected to the control board via a serial interface.

A typical scheme for using the MC3PHAC microcontroller for frequency control of a three-phase motor is shown in Fig.1.

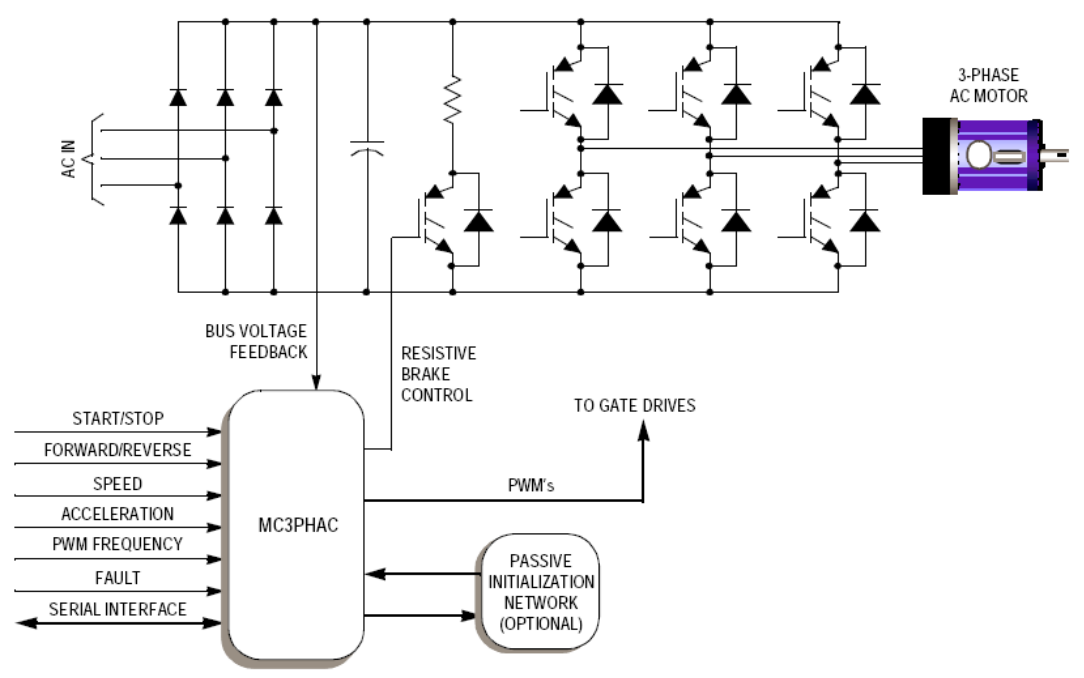

Fig. 1. Basic diagram of MC3PHAC microcontroller application 
The "Start" or "Stop" modes are set by the signal level at the START pin, and the direction of rotation of the BP shaft is determined by the signal level at the FWD pin. The speed and acceleration are set at the analog inputs SPEED and ACCEL, respectively. The PWM frequency of the internal oscillator depends on the voltage at the PWMFREQ_RxD pin. A serial interface (SERIAL INTERFACE) is provided for programmatically setting the constants on the RETRY_TxD and PWMFREQ_RxD pins. Optionally, it is possible to set parameters via a local network.

If the signal at the VBOOST_MODE pin is high, the microcontroller (MC) goes into stand-alone mode. In this mode, the functionality of several MC pins is changed so that the device can control the motor without requiring setup information from an external computer. In this case, several parameters are set as an analog voltage at the corresponding pin.

In the configuration of the stand-alone mode of the MC (Figure 2), the values of speed and acceleration are set by trimming resistors [2 - 4]. The base frequency $(50 \mathrm{~Hz}$ or $60 \mathrm{~Hz})$ and the PWM polarity are set by the position of the jumper JP1, 2, 3, 4. System reset RESET is carried out by the RC-chain when turned on or by the corresponding button during operation. The internal clock is set by an external crystal element. Analog (A) and digital power and ground circuits are separate.

The Dead Time characterizes the pause between signals supplied to the vertical of the power switches to prevent through currents.

The Retry Time after the elimination of the cause that caused the error signal determines the restoration of normal operation of the MC.

The voltage addition in BOOST mode allows compensate for engine power losses at low frequencies.

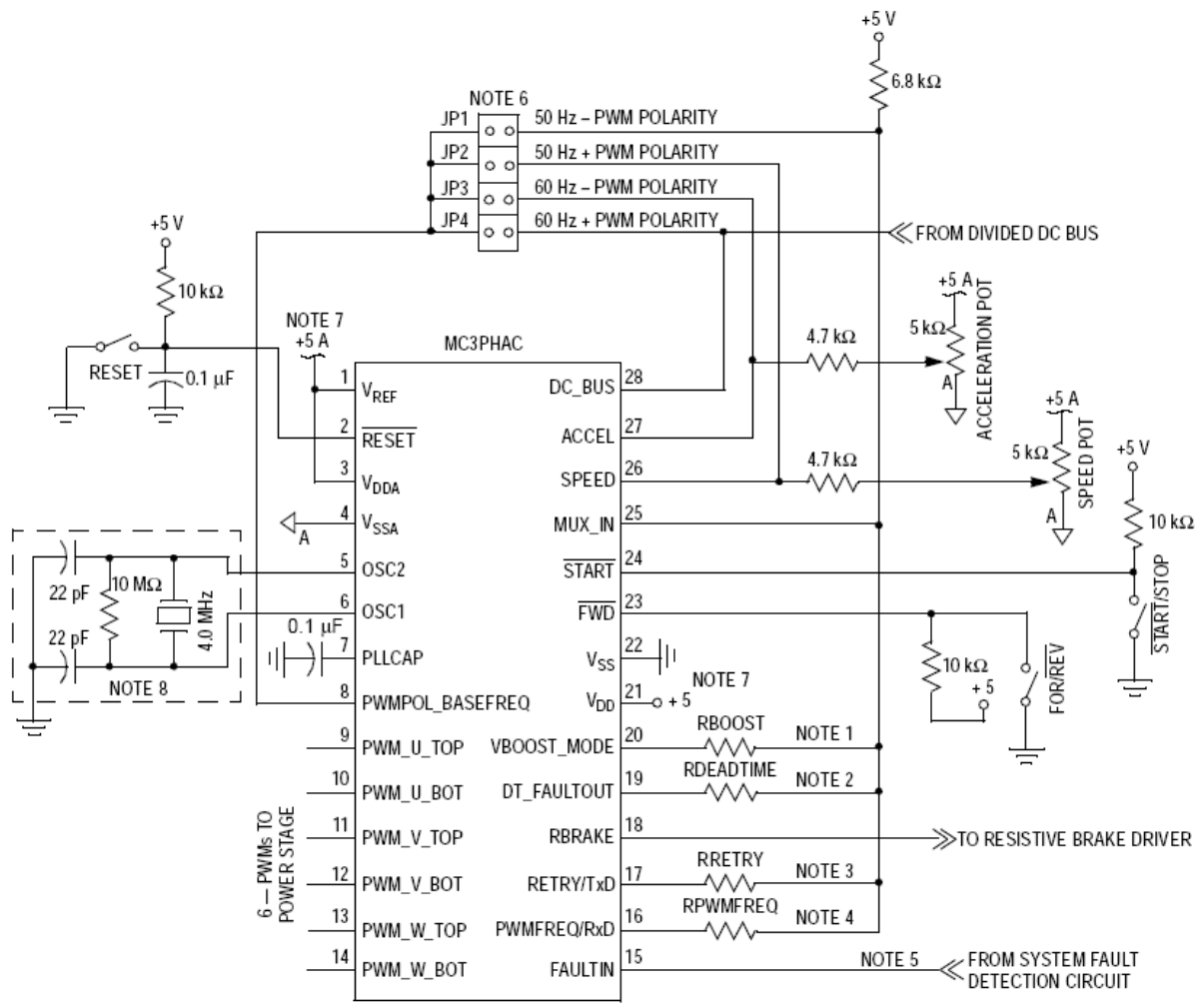

Fig. 2. Configuration of MC3PHAC microcontroller in stand-alone mode 


\subsection{Three-phase driver}

The development of the inverter layout was preceded by experimental studies of the technical capabilities of the MC3PHAC microcontroller (and its modifications) and threephase IR2130 drivers, IR2131 and IR2132 from International Rectifier with non-rated operating modes of the latter [5 - 7]. Each of the nodes was designed in the form of a separate breadboard, in which panels were provided for quick replacement of the main microcircuits. The piping of the microcontroller circuit basically corresponded to the typical one for an autonomous mode of operation. However, to reduce the dynamic losses on the keys, an attempt was made to reduce the synchronization frequency of the microcontroller and, as a result, the frequency of pulse-width modulation of control signals.

IR213x - high-voltage, high-speed drivers for MOS transistors and IGBT transistors with separate output control channels for the keys of the upper and lower levels (Figure 3).

The logic inputs of the microcircuit are compatible with 5V CMOS or LSTTL outputs. The current interrupt function, acting on all 6 outputs, uses the signal from the resistor that controls the current. The shutdown input is used by the configured shutdown function. An open drain signal FAULT indicates any shutdown reason. The output drivers are characterized by a high impulse current of the buffer stage, which is done to minimize the transverse conductance of the drivers. Propagation times are matched for ease of use at high frequencies. The output channels are designed to drive n-channel MOS transistors or IGBT transistors, including those used as top-level switches with operating voltages up to $600 \mathrm{~V}$ and higher [7 - 9].

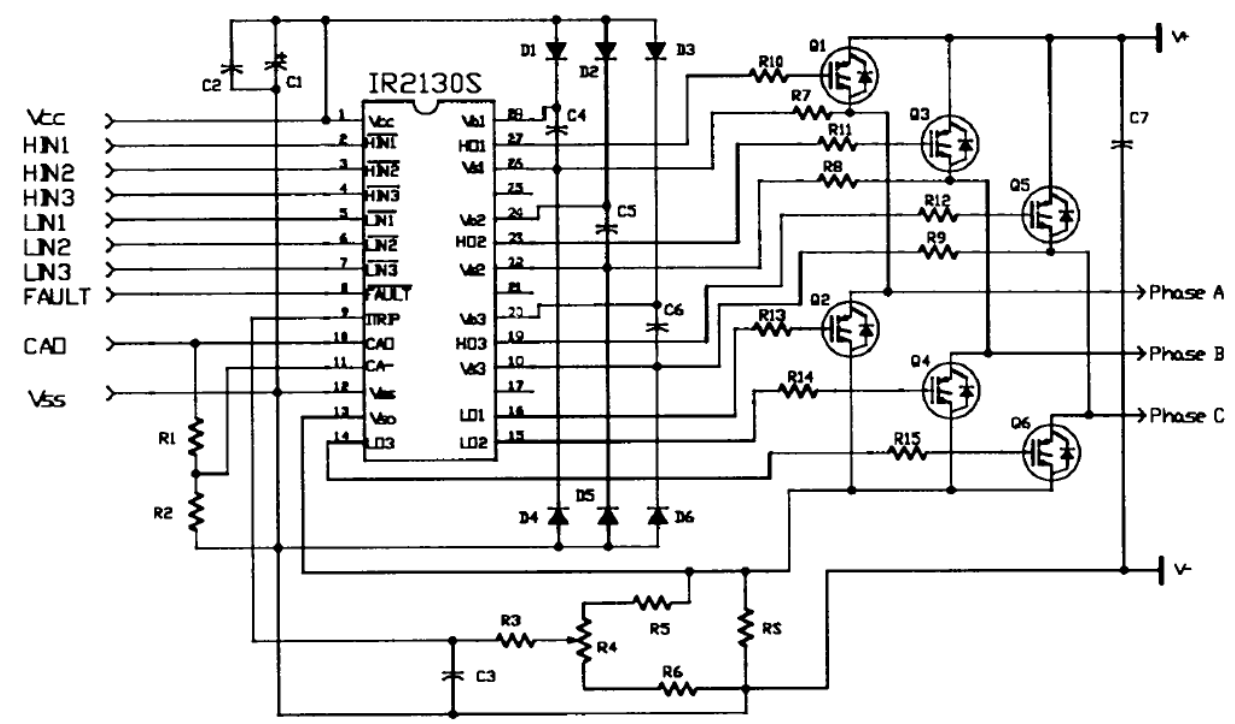

Fig. 3. Output circuit diagram of the IR2130S driver

\subsection{Bridge power transistors}

As the output transistors of the three-phase bridge (see Figure 3), n-MOS devices with a STB55NF06 reverse diode from STMicroelectronics, having a typical resistance drainsource in the open state Rds (on) $=0.015 \mathrm{Ohm}$ at the maximum drain current $\mathrm{Id}=50 \mathrm{~A}$ and the maximum drain-source voltage in the closed state Vdss $=60 \mathrm{~V}$. The working 
temperature range is from $-55^{\circ} \mathrm{C}$ to $175^{\circ} \mathrm{C}$. Dissipated power $110 \mathrm{~W}$ at a temperature of $25^{\circ} \mathrm{C}[10]$.

During the circuit design, computer simulation was carried out by means of the MicroCap program [11] of the output half-bridge according to a circuit like Figure 3. In this case, we used SPICE-models of field-effect transistors IRFZ44N and IRFZ48N, developed by International Rectifier (analogs of STB55NF06). The use of $200 \mathrm{Ohm}$ gate resistors R10 R15 showed that under these conditions the transistors operate with a noticeable through current, which disappears if the resistor values are reduced to $100 \mathrm{Ohm}$. The presence of gate resistors prevents the driver from latching on, but at the same time increases the turnoff time of the transistors. To eliminate this undesirable effect, bypass diodes were used to bypass the gate resistors. This made it possible to significantly reduce the dynamic losses when turning off the MOS transistors.

Among other ways to prevent latching, it should be noted snubber - a damping circuit of a series-connected resistor and capacitor, installed between the output of the transistor halfbridge and one of the poles of its power supply.

Contrary to conventional wisdom, the dynamic properties of a MOS transistor are most accurately characterized not by the values of its parasitic capacitances, but by the total gate charge Qg. This important value relates the gate pulse current to the switching time of the transistor, making it possible to correctly calculate the control unit. At a constant gatesource voltage, the gate charge decreases with increasing drain current and with decreasing drain-source voltage [12 - 15].

These technical solutions made it possible to reduce the current consumed by the power circuits of the low-voltage inverter by an average of $5 \%$.

\section{Model of the inverter}

The result of the generalization of the research was the developed and manufactured model of the inverter for controlling a three-phase asynchronous motor.

The composition of the layout is quite universal. This is where the MC itself, the threephase driver and power transistors of the three-phase bridge, snubbers and supply voltage converter circuits are located.

The schematic diagram of the breadboard is shown in Figure 4. The breadboard is powered by a direct current source with a voltage of $18-32 \mathrm{~V}$, the role of which was played by a pulsed power supply KR400A-220 S24-4 with an output voltage of $24 \mathrm{~V}$, as well as a laboratory power supply unit with an adjustable voltage.

Through the filter C6, L2, C7, the main supply voltage is supplied directly to the power switches of the three-phase bridge VT2-VT7 and the switch of the resistive (R40) brake VT8, controlled by the RBRAKE signal of the microcontroller. Power supply for 5V and $15 \mathrm{~V}$ chips is formed by integral stabilizers DA1 and DA2.

Three snubbers at the output of the bridge C20 / R41, C22 / R43, C21 / R42, as well as filters L3-L5 perform damping functions in relation to the three-phase bridge transistors. Resistor R38 is used as a sensor for the current protection circuit without elements of adjustment of the protection operation level for the sake of simplicity. Divider resistors R28, R35 generate a DS_BUS signal (nominal 3.5V) for monitoring the voltage continuity of the power bus for the microcontroller [7].

The binding of the DD1 microcontroller circuit basically corresponds to the typical one for stand-alone operation. Additionally, damping capacitors C24-C27, microcontroller ADC power filter L1, C4, DD2 and DD3 optocouplers, VT1 error signal key is introduced. The latter matches the error signal at the output of the FAULT driver with the FAULTIN input of the microcontroller, simultaneously indicating it with the HL1 LED. 


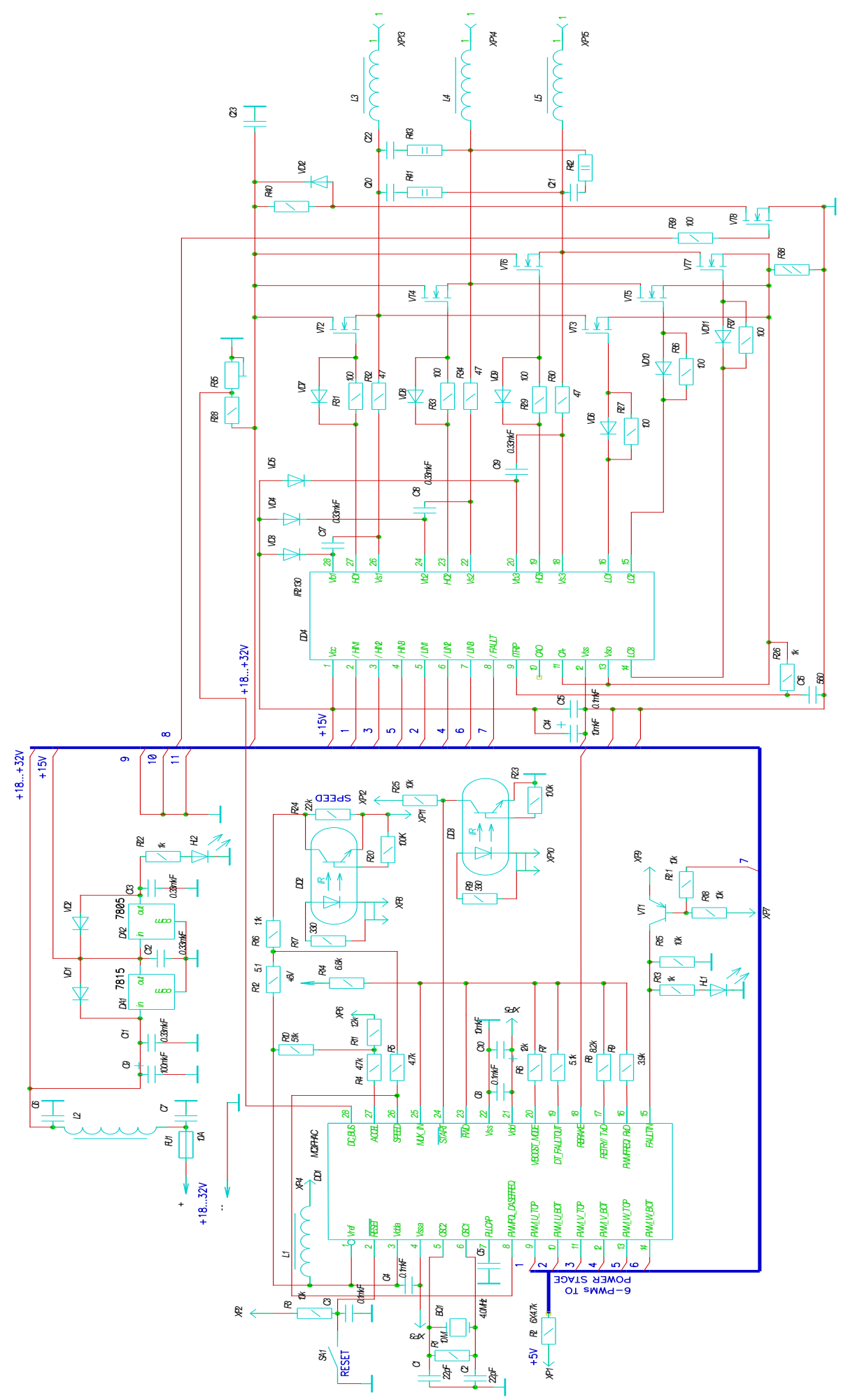

Fig. 4. Schematic electrical diagram of the inverter 
Optocouplers provide galvanic isolation of external speed control circuits (DD2) and motor start and stop modes (DD3). To set two rated speeds of the blood pressure, trimmer resistors R16, R24 are used. The fixed value of acceleration and deceleration is determined by the ratio of the resistances of the resistors $\mathrm{R} 4$ and $\mathrm{R} 11$.

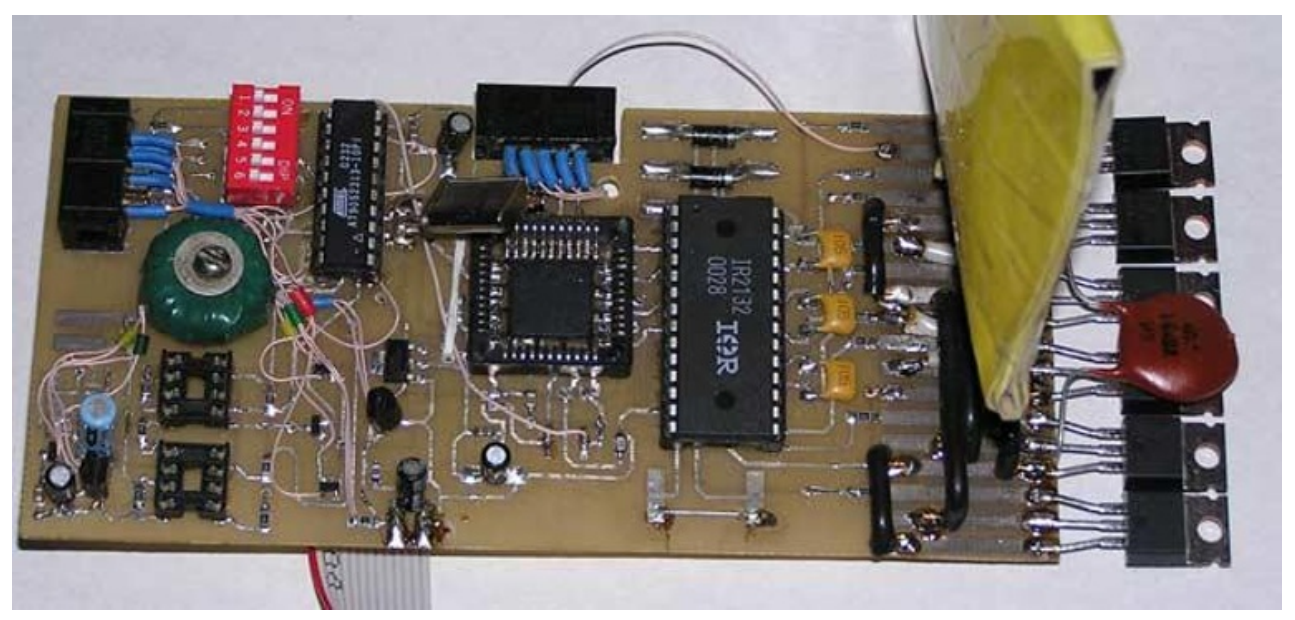

Fig. 5. Model of the inverter

The model of the inverter (see Figure 5) was studied at the laboratory bench, consisting of a 100W AIR56A4U3 three-phase asynchronous electric motor (specially converted for these purposes for a low supply voltage for the manufacturer) and a DC generator, the shafts of which were connected by a coupling, as well as the electrical load of the generator in the form of resistors. An induction motor drove a generator into rotation, which served as a variable mechanical load on the motor shaft. The change in the braking torque was created in the generator by changing the generator armature current using load resistors. Investigations of the engine operation were carried out both in the "star" connection scheme and in the "triangle" connection scheme.

\section{Conclusion}

The features of the design of an asynchronous frequency-controlled three-phase electric driver with a low-voltage autonomous DC power supply, are presented. All these shaped the basis for the creation of an operating model of an electric driver inverter and components selection. The results of the simulation of inverter power circuits in the MicroCap program made it possible to reduce the inverter consumption current by an average of $5 \%$. Reducing energy losses is achieved by reducing dynamic losses in the power circuits of a low-voltage inverter and using several circuit solutions.

\section{References}

1. A. Usoltsev, Frequency control of asynchronous motors ITMO (2006)

2. MC3PHAC Monolithic Intelligent Motor Controller, https://www.nxp.com/docsen/data-sheet/MC3PHAC.pdf 
3. General-Purpose 3-Phase AC Industrial Motor Controller Reference Design. Designer Reference Manual, http: www.motorola.com/mcu

4. D. Nazarenko, https://www.motorjock.com/mc3phac-info.html

5. Six-Output 600V MGDs Simplify 3-Phase Motor Drives, http://www.irf.com/technical-info/appnotes/an-985.pdf

6. Maximizing the Latch Immunity of the IR2151 \& IR2152 in Ballast Applications, http://www.irf.com/technical-info/designtp/dt94-9.pdf

7. Electronic Components Datasheet Search, https://www.alldatasheet.com/datasheetpdf/pdf/68069/IRF/IR2130.html

8. J. Adams, Bootstrap Component Selection for Control IC's, http://www.irf.com/technical-info/designtp/dt98-2.pdf

9. Use Gate Charge to Design the Gate drive Circuit for Power MOSFETs and IGBTs, Application Note AN-944, http://www.irf.com/technical-info/appnotes/an-944.pdf

10. N-Channel Power MOSFET STB55NF06, http:www.st.com

11. Micro-Cap 12 Electronic Circuit Analysis Program Reference Manual Copyright 1982-2018 by Spectrum Software 1021 South Wolfe Road Sunnyvale, CA 94086, https://www.spectrum-soft.com/download/rm12.pdf

12. A. Boldyrev, I. Semko, A. Chubukin, Designing drives for stationary and mobile devices based on pulse-width converters (2007)

13. UCC3807-1 Programmable Maximum Duty Cycle PWM Controller, http://www.ti.com/general/docs/lit/getliterature.tsp?genericPartNumber=ucc3807$1 \&$ fileType $=$ pdf

14. Modelling, analysis and compensation of the current-mode converter, http://www.thierry-lequeu.fr/data/SLUA101.pdf

15. W. Lei, T. Man, A General Approach for Optimizing Dynamic Response for Buck Converter, https://www.onsemi.com/ pub/Collateral/AND8143-D. pdf 